${ }^{1}$ Diagnostics for the Real World (Europe) Ltd, Cambridge Science Park, Cambridge CB4 OWG

${ }^{2}$ University of Cambridge, Department of Haematology, Diagnostics Development Unit, EABC Site, Long Road,

Cambridge CB2 2PT

${ }^{3}$ Sexually Transmitted Bacteria Reference Laboratory, Health Protection Agency Centre for Infections, London NW9 5HT

${ }^{4}$ Brook in Birmingham, 56-61 John Bright Street, Birmingham B1 1BL

${ }_{5}^{5}$ Barts and The London NHS Trust, London E1 1BB

Correspondence to: $\mathrm{H} \mathrm{H}$ Lee hl207@cam.ac.uk

doi:10.1136/bmj.39402.463854.AE

\title{
New point of care Chlamydia Rapid Test—bridging the gap between diagnosis and treatment: performance evaluation study
}

\author{
Lourdes Mahilum-Tapay, director of scientific affairs, ${ }^{1}$ Vivian Laitila, director of regulatory affairs, ${ }^{1}$ \\ James I Wawrzyniak, research assistant, ${ }^{2}$ Sarah Alexander, clinical scientist, ${ }^{3}$ Alison Swain, senior doctor, ${ }^{4}$ \\ Penelope Barber, chief executive officer, ${ }^{4}$ Ines Ushiro-Lumb, virologist, ${ }^{5}$ Beng T Goh, consultant, \\ genitourinary medicine clinics, ${ }^{5}$ Catherine Ison, director, ${ }^{3}$ Helen $\mathrm{H}$ Lee, reader in medical biotechnology ${ }^{2}$
}

\section{ABSTRACT}

Objective To evaluate the performance of a new Chlamydia Rapid Test with vaginal swab specimens as a potential tool for chlamydia diagnosis and screening. Design Performance evaluation study.

Settings A young people's sexual health centre (site 1) and two genitourinary medicine clinics (sites 2 and 3 ) in the United Kingdom.

Participants 1349 women aged between 16 and 54 attending one of the three clinics.

Main outcome measures Sensitivity, specificity, positive predictive value, and negative predictive value of the Chlamydia Rapid Test versus polymerase chain reaction and strand displacement amplification assays; correlation between the Chlamydia Rapid Test visual signal and organism load; acceptability to participants of self collected vaginal swabs as the specimen type for Chlamydia testing.

Results Polymerase chain reaction positivity rates for Chlamydia trachomatis infection were $8.4 \%(56 / 663)$ at site $1,9.4 \%(36 / 385)$ at site 2 , and $6.0 \%(18 / 301)$ at site 3. Compared with polymerase chain reaction assay, the resolved sensitivity, specificity, positive predictive value, and negative predictive value of the Chlamydia Rapid Test were $83.5 \%$ (91/109), 98.9\% (1224/1238), 86.7\% (91/105), and 98.6\% (1224/1242). Compared with strand displacement amplification assay, sensitivity and specificity of the Chlamydia Rapid Test were $81.6 \%$ (40/49) and $98.3 \%(578 / 588)$. Organism load of self collected vaginal swabs ranged from $5.97 \times 10^{2}$ to $1.09 \times 10^{9}$ Chlamydia plasmids per swab, which correlated well with the Chlamydia Rapid Test's visual signal $(r=0.6435, \mathrm{P}<0.0001)$. Most (95.9\%) surveyed participants felt comfortable about collecting their own swabs.

Conclusions The performance of the Chlamydia Rapid Test with self collected vaginal swabs indicates that it would be an effective same day diagnostic and screening tool for Chlamydia infection in women. The availability of Chlamydia Rapid Test results within 30 minutes allows for immediate treatment and contact tracing, potentially reducing the risks of persistent infection and onward transmission. It could also provide a simple and reliable alternative to nucleic acid amplification tests in chlamydia screening programmes.

\section{INTRODUCTION}

Chlamydia trachomatis infection is the most prevalent sexually transmitted bacterial infection worldwide. It is common among sexually active young women and, especially if left undiagnosed and untreated, can result in complications such as pelvic inflammatory disease, ectopic pregnancy, and infertility. ${ }^{12}$ In the absence of an effective screening programme, chlamydial infection often remains undetected, given that up to $80 \%$ of infected women have no symptoms. ${ }^{3}$ Developed countries such as the United Kingdom have national screening programmes in place, in which almost all specimens are tested by nucleic acid amplification tests and most women provide non-invasive specimens, such as first void urine and vulvovaginal swabs, for analysis. ${ }^{4}$ In contrast, screening programmes for Chlamydia are almost non-existent in the developing world, where Chlamydia testing is not done routinely, even among high risk populations such as female sex workers. ${ }^{5-7}$ Economic constraints as well as the lack of simple and rapid tests that are instrument independent and easy to do are major obstacles to such routine screening. Consequently, diagnosis and treatment of chlamydial infection are based on syndromic management principles that have poor specificity for chlamydial infection in women. ${ }^{89}$

Currently available rapid tests for Chlamydia lack sensitivity and are not licensed for vaginal swab specimens, with the exception of the Handi-Lab test (Zonda, Moraga, CA), which has the Conformité Européenne mark. A recent Norwegian study assessing the performance of this test in women reported values of only $25 \%$ for sensitivity, $80.6 \%$ for specificity, and $23.5 \%$ for positive predictive value. ${ }^{10}$

We evaluated the performance of the Chlamydia Rapid Test, a new assay developed at the Diagnostics Development Unit, University of Cambridge. This assay was devised to aid in the diagnosis of chlamydial 


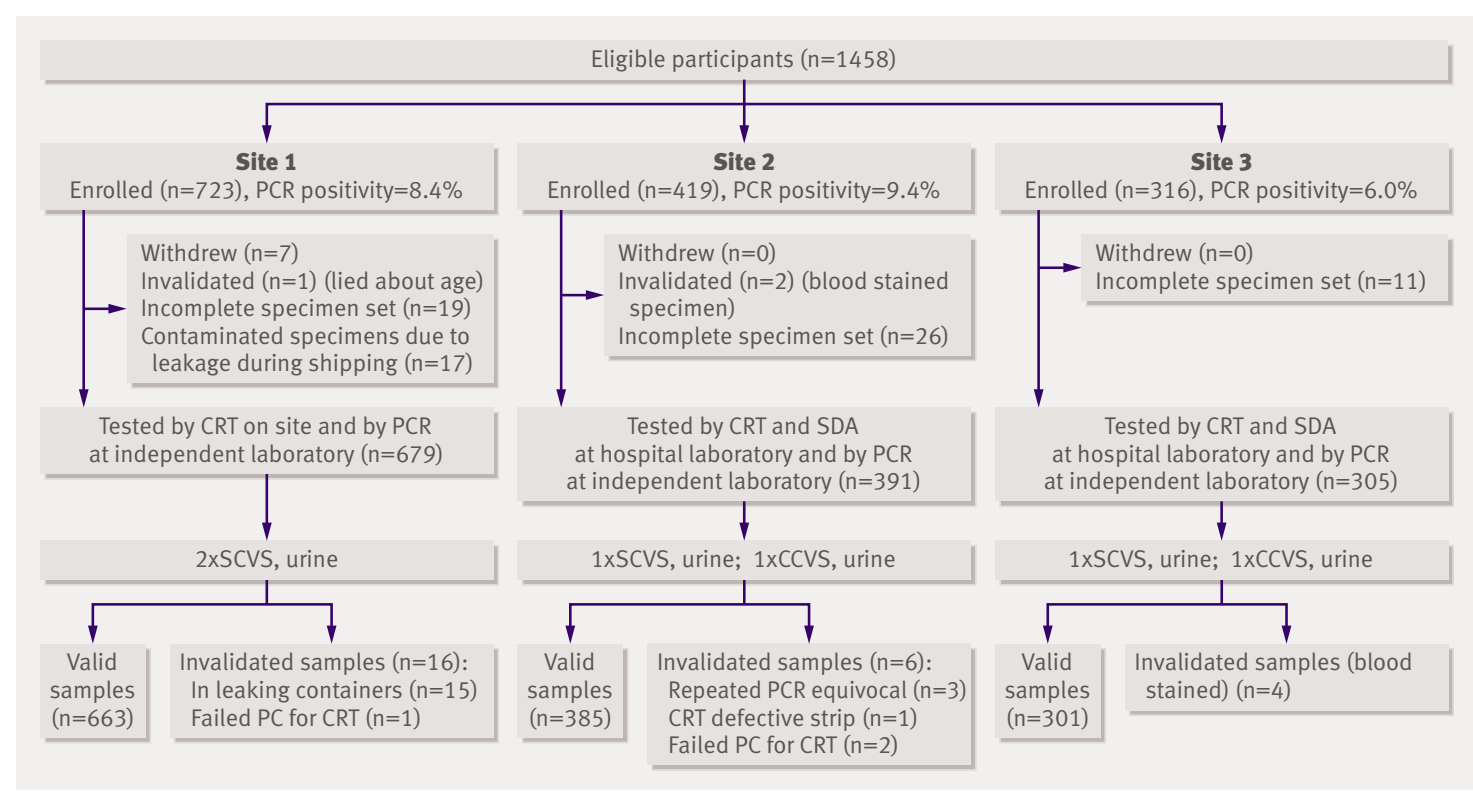

Fig 1 | Recruitment and testing algorithm for study participants. CCVS=clinician collected vaginal swab specimens; CRT=Chlamydia Rapid Test; $P C=$ procedural control; $P C R=$ polymerase chain reaction; $S D A=$ strand displacement amplification assay; SCVS=self collected vaginal swab specimens

infection and to provide a screening tool for Chlamydia that can be used with vaginal swab specimens in resource limited settings.

\section{METHODS}

Sites

We selected a young people's sexual health centre (Brook in Birmingham, site 1) and two genitourinary medicine clinics (Ambrose King Centre, site 2, and Barts Sexual Health Centre, site 3) in the UK as evaluation sites for the Chlamydia Rapid Test. The study ran from November 2005 to March 2006.

\section{Participants}

We invited all women attending the three sites to join the study. We considered them to be eligible if they were at least 16 years old, had not taken antibiotics in the previous month, were not menstruating at the time of their visit, and were able to understand the written information forms for the study. We gave each participant a patient information sheet about the study. Participants gave written informed consent and were then interviewed confidentially about their symptoms and relevant sexual history. After collection of clinical specimens, we surveyed the participants with a written questionnaire concerning sample collection methods and preferences.

\section{Specimen collection}

At site 1, each participant provided two self collected vaginal swabs and a first void urine specimen, as clinicians did not routinely examine every attender at this site. At sites 2 and 3, each participant provided one clinician collected vaginal swab, one self collected vaginal swab, and one first void urine specimen. In addition, we collected a routine endocervical swab specimen for Chlamydia testing with the ProbeTec ET strand displacement amplification assay (BectonDickinson Diagnostic Systems, Sparks, MD), the test used at the genitourinary medicine clinics.

Each participant was shown an illustrated instruction sheet detailing collection of vaginal swab specimens before she was given the swab kit. First void urine specimens at all sites were collected at least two hours after the participant had last voided. We divided urine specimens into two portions, one for polymerase chain reaction testing by an independent laboratory and the other for freezing and storage in case testing of discordant samples by the Aptima Combo 2 transcription mediated amplification assay at the Sexually Transmitted Bacteria Reference Laboratory, Health Protection Agency, Colindale, was needed. We handled and stored all specimens according to the recommendations of the relevant test manufacturers. An independent clinical laboratory evaluated the reproducibility of the Chlamydia Rapid Test in accordance with the National Committee on Clinical Laboratory Standards' guideline. ${ }^{11}$ Two operators tested randomised, masked 10 member panels in duplicate, over a period of five days, following the procedure for the Chlamydia Rapid Test.

Testing of vaginal swabs with Chlamydia Rapid Test Clinic staff tested vaginal swabs on site; all staff had passed testers' requirements in accordance with the National Committee on Clinical Laboratory Standards. ${ }^{11}$ We subjected each swab to extraction by sequential addition of $400 \mu \mathrm{l}$ of reagent $1,300 \mu \mathrm{l}$ of reagent 2 , and $100 \mu \mathrm{l}$ of reagent 3 to the swab in a tapered sample preparation tube, with gentle mixing 
Table 1 Unresolved performance of Chlamydia Rapid Test with self collected vaginal swab specimens versus polymerase chain reaction. Values are percentages (numbers) $(95 \%$ confidence intervals)

\begin{tabular}{|c|c|c|c|c|}
\hline Site & Sensitivity & Specificity & Positive predictive value & Negative predictive value \\
\hline $1(n=663)$ & $83.9(47 / 56)(74.3$ to 93.5$)$ & $98.8(600 / 607)(98.0$ to 99.7$)$ & $87.0(47 / 54)(78.1$ to 96.0$)$ & $98.5(600 / 609)$ (97.6 to 99.5$)$ \\
\hline $2(n=385)$ & $80.6(29 / 36)(67.6$ to 93.5$)$ & $98.0(342 / 349)(96.5$ to 99.5$)$ & $80.6(29 / 36)(67.6$ to 93.5$)$ & $98.0(342 / 349)(96.5$ to 99.5$)$ \\
\hline $3(n=301)$ & $83.3(15 / 18)(66.1$ to 100$)$ & $99.6(282 / 283)(99.0$ to 100$)$ & $93.8(15 / 16)(81.9$ to 100$)$ & $98.9(282 / 285)(97.8$ to 100$)$ \\
\hline Total $(n=1349)$ & $82.7(91 / 110)(75.7$ to 89.8$)$ & $98.8(1224 / 1239)$ (98.2 to 99.4$)$ & $85.8(91 / 106)(79.2$ to 92.5$)$ & $98.5(1224 / 1243)$ (97.8 to 99.2$)$ \\
\hline
\end{tabular}

No significant difference in Chlamydia Rapid Test performance was apparent among three sites ( $\mathrm{P}=0.278, \mathrm{k}$ statistics).

by hand between additions. The sample preparation reagents were supplied in unit doses, eliminating the need for precise pipetting. We then capped the extraction tube and used it as a dropper to deliver five drops (approximately $100 \mu \mathrm{l}$ ) of the extracted sample to a tube containing the lyophilised amplification and detection reagents. We gently mixed the resulting mixture until it became a clear pink solution, after which we added the test strip, coated with a monoclonal antibody to chlamydial lipopolysaccharide ${ }^{12}$ and including a procedural control, to the solution and allowed it to stand for 25 minutes before reading the result. To ensure that the target antigen (chlamydial lipopolysaccharide) was extracted optimally from the viscous vaginal swab sample, we repeated the entire procedure on each swab. The appearance of a result line on either or both test strips indicated the presence of Chlamydia.

Testing of specimens by polymerase chain reaction and transcription mediated assay

We sent urine specimens to a laboratory accredited by the UK Accreditation Service for testing for Chlamydia trachomatis with the Amplicor Chlamydia trachomatis polymerase chain reaction assay (Roche Diagnostic Systems, Branchburg, NJ). Samples that yielded discordant results between the Chlamydia Rapid Test and the polymerase chain reaction assay were tested by transcription mediated assay at the Sexually Transmitted Bacteria Reference Laboratory. In addition, 100 of the total number of polymerase chain reaction negative specimens and 20 of the concordant positive samples were also randomly tested by this assay to minimise potential bias introduced by testing discordant samples only.

\section{Quantification of organism load}

We did real time quantitative polymerase chain reaction analysis as described previously, ${ }^{13}$ with minor modifications. ${ }^{14}$ We placed the second of the two self collected vaginal swabs collected at site 1 for polymerase chain reaction positive women into M4RT medium (Remel, Lenexa, KS) and incubated it at $37^{\circ}$ $\mathrm{C}$ for 30 minutes before thorough agitation with a vortex mixer to release the vaginal fluid from the swab. We centrifuged a portion $(500 \mu \mathrm{l})$ of the resulting extract at $17860 \times g$ for 15 minutes at $25^{\circ} \mathrm{C}$ (Megafuge 1.0R; Hereaus, Osterode, Germany) and processed it as described previously. ${ }^{14}$ We used a $20 \mu \mathrm{l}$ portion of each DNA extract for quantitative polymerase chain reaction analysis.

\section{Statistical analysis}

We used standard statistical methods to analyse data with SAS V9.1 software.

\section{RESULTS}

\section{Participants and sites}

A total of 1349 participants at three clinical sites contributed samples to the multicentre performance evaluation of the Chlamydia Rapid Test. The mean age of participants was 18.5 (range 16-27.4) years at site 1, 25.4 (16-49.7) years at site 2 , and 27.8 (17.1-54.8) years at site $3(\mathrm{P}<0.0001$ for each comparison). We compared clinician collected and self collected vaginal swab specimens at two clinical sites, and we compared self collected vaginal swab specimens with polymerase chain reaction results for first void urine at all sites. Figure 1 summarises the recruitment and testing algorithm for the study participants at all clinical sites.

Most participants from site 1 attended the centre for contraception and other reproductive health services and were asymptomatic. In contrast, about $67 \%$ (441/ 662 ) of the participants from the genitourinary medicine clinics presented with symptoms that included vaginal discharge $(305 / 662,46 \%)$ and lower abdominal pain $(149 / 657,23 \%)$. In addition, 3\% (23/668) were diagnosed as having pelvic inflammatory disease.

\section{Reproducibility testing for Chlamydia Rapid Test}

We found 100\% concordance between the expected results and the results generated from randomised and masked panels by testers using the Chlamydia

Table 2 | Performance of Chlamydia Rapid Test with self collected or clinician collected vaginal swab specimens from participants at sites 2 and 3 versus polymerase chain reaction. Values are percentages (numbers) ( $95 \%$ confidence intervals)

\begin{tabular}{|c|c|c|c|c|}
\hline Sample type & Sensitivity & Specificity & Positive predictive value & Negative predictive value \\
\hline Self collected vaginal swab $(n=686)$ & $81.5(44 / 54)$ (71.1 to 91.8$)$ & $98.7(624 / 632)$ (97.9 to 99.6$)$ & $84.6(44 / 52)(74.8$ to 94.4$)$ & $98.4(624 / 634)(97.5$ to 99.4$)$ \\
\hline Clinician collected vaginal swab $(n=686)$ & $77.8(42 / 54)(66.7$ to 88.9$)$ & $99.2(627 / 632)(98.5$ to 99.9$)$ & $89.4(42 / 47)(80.5$ to 98.2$)$ & $98.1(627 / 639)(97.1$ to 99.2$)$ \\
\hline
\end{tabular}

Differences between self collected and clinician collected vaginal swab specimens were not significant by McNemar's test $(P=0.096)$. 
Table 3 | Resolved sensitivity, specificity, positive predictive values, and negative predictive values of Chlamydia Rapid Test with self collected vaginal swab specimens after testing of discordant samples by Sexually Transmitted Bacteria Reference Laboratory. Values are percentages (numbers) ( $95 \%$ confidence intervals)

\begin{tabular}{|c|c|c|c|c|}
\hline Site & Sensitivity & Specificity & Positive predictive value & Negative predictive value \\
\hline $1(n=663)$ & $85.5(47 / 55)(76.1$ to 94.8$)$ & $99.0(600 / 606)(98.2$ to 99.8$)$ & $88.7(47 / 53)(80.1$ to 97.2$)$ & $98.7(600 / 608)(97.8$ to 99.6 \\
\hline $2(n=385)$ & $80.6(29 / 36)(67.6$ to 93.5$)$ & $98.0(342 / 349)(96.5$ to 99.5$)$ & $80.6(29 / 36)(67.6$ to 93.5$)$ & $98.0(342 / 349)(96.5$ to 99.5$)$ \\
\hline $3(n=301)$ & $83.3(15 / 18)(66.1$ to 100$)$ & $99.6(282 / 283)(99.0$ to 100$)$ & $93.8(15 / 16)(81.9$ to 100$)$ & $99.0(282 / 285)(97.8$ to 100$)$ \\
\hline Total $(n=1349)$ & $83.5(91 / 109)(76.5$ to 90.5$)$ & $98.9(1224 / 1238)(98.3$ to 99.5$)$ & $86.7(91 / 105)(80.2$ to 93.2$)$ & $98.6(1224 / 1242)$ (97.9 to 99.2$)$ \\
\hline
\end{tabular}

Rapid Test. A previous study in which a modified version of the Chlamydia Rapid Test was used for trachoma testing by four novice operators in Tanzania also showed excellent reproducibility. ${ }^{12}$

Specimen choice for polymerase chain reaction and strand displacement amplification assay testing

We assessed the performance of the Chlamydia Rapid Test in order to meet the requirements for Conformité Européenne licensure, which stipulate that the comparator test should be a "state of the art" assay and use specimens approved for the test. Participants from site 1 did not provide endocervical swabs, preventing the pooling of data from all three sites. Given this condition, we chose polymerase chain reaction testing, which is licensed for both urine and endocervical specimens, as the "gold standard" for the study. Studies of Chlamydia trachomatis polymerase chain reaction testing have shown equal performance with cervical and urine specimens, ${ }^{15}$ across all volumes of urine tested $(<20-90 \mathrm{ml}),{ }^{16}$ and good reproducibility. ${ }^{17}$ For the genitourinary medicine clinics, endocervical specimens were additionally collected by the clinician and were tested by strand displacement amplification assay at the hospital laboratory.

\section{Performance of Chlamydia Rapid Test}

The Chlamydia Rapid Test is an immunoassay based test that detects chlamydial lipopolysaccharide. ${ }^{12} \mathrm{We}$ used Chlamydia Rapid Test assay with self collected vaginal swab specimens (all sites) or clinician collected vaginal swab specimens (sites 2 and 3), whereas we used Chlamydia trachomatis polymerase chain reaction testing with first void urine specimens collected at all clinical sites. We compared the performance of the Chlamydia Rapid Test between self collected and clinician collected specimens at sites 2 and 3.

Positivity rates for polymerase chain reaction were $8.4 \%(56 / 663)$ at site $1,9.4 \%(36 / 385)$ at site 2 , and $6.0 \%(18 / 301)$ at site 3 . For self collected vaginal swab specimens, unresolved Chlamydia Rapid Test sensitivity and specificity across all three sites were $82.7 \%$ and $98.8 \%$ (table 1 ). We found no significant difference in the performance of the Chlamydia Rapid Test among clinical sites $(\mathrm{P}=0.278)$. The combined data from the genitourinary medicine clinics showed the unresolved Chlamydia Rapid Test sensitivity and specificity to be $81.5 \%$ and $98.7 \%$ with self collected vaginal swab specimens and $77.8 \%$ and $99.2 \%$ with clinician collected vaginal swab specimens (table 2). After testing of discordant samples by the Sexually Transmitted Bacteria Reference Laboratory, the Chla mydia Rapid Test had an overall sensitivity and specificity across all clinical sites of $83.5 \%$ and $98.9 \%$ (table 3). All 100 randomly selected polymerase chain reaction negative samples were confirmed as negative by transcription mediated amplification testing, whereas one out of the 20 concordant positive samples tested showed an equivocal result by transcription mediated amplification assay.

The Chlamydia Rapid Test had an overall unresolved positive predictive value of $85.8 \%$ and negative predictive value of $98.5 \%$ with self collected vaginal swab specimens (table 1). After testing of discordant samples, the resolved positive predictive value was $86.7 \%$ and the negative predictive value was $98.6 \%$ (table 3 ). The positive predictive value and negative predictive value of Chlamydia Rapid Test with clinician collected vaginal swab specimens at the genitourinary medicine clinics were $89.4 \%$ and $98.1 \%$, and the corresponding values for self collected vaginal swab specimens were $84.6 \%$ and $98.4 \%$ (table 2). Chlamydia Rapid Test assay of self collected vaginal swab specimens at the site with the lowest Chlamydia positivity rate yielded a positive predictive value of $93.8 \%$ and a negative predictive value of $98.9 \%$ (table 1). The difference in performance of the Chlamydia Rapid Test between self collected and clinician collected vaginal swab specimens was not sig nificant $(\mathrm{P}=0.096)$.

Comparison of the Chlamydia Rapid Test with strand displacement amplification assay using endocervical swab specimens from participants at the genitourinary

Table 4 | Summary of Chlamydia Rapid Test performance with self collected vaginal swab specimens versus polymerase chain reaction with first void urine or strand displacement amplification assay with endocervical swabs for sites 2 and 3 . Values are percentages (numbers) ( $95 \%$ confidence intervals)

\begin{tabular}{|c|c|c|c|c|}
\hline Comparator test & Sensitivity & Specificity & Positive predictive value & Negative predictive value \\
\hline Polymerase chain reaction $(n=686)$ & $81.5(44 / 54)(71.1$ to 91.8$)$ & $98.7(624 / 632)(97.9$ to 99.6$)$ & $84.6(44 / 52)(74.8$ to 94.4$)$ & $98.4(624 / 634)(97.5$ to 99.4$)$ \\
\hline Strand displacement assay $(n=637)$ & $81.6(40 / 49)(70.8$ to 92.5$)$ & $98.3(578 / 588)(97.2$ to 99.3$)$ & $80.0(40 / 50)(68.9$ to 91.1$)$ & $98.8(578 / 585)(97.9$ to 99.7$)$ \\
\hline
\end{tabular}

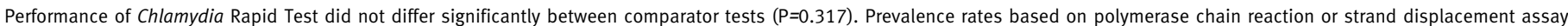
data did not differ significantly by McNemar's test for correlated proportions $(P=0.739$ ). 


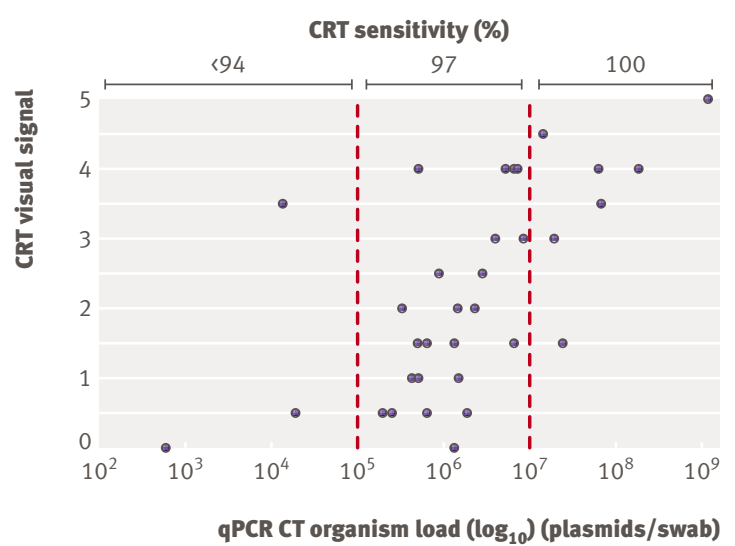

Fig 2 |Chlamydia trachomatis (CT) organism load by quantitative polymerase chain reaction (qPCR; plasmids/ swab) $(\mathrm{n}=33, r=0.644, \mathrm{P}<0.0001) . \mathrm{CRT}=$ Chlamydia Rapid Test

medicine clinics yielded an overall sensitivity of $81.6 \%$ and specificity of $98.3 \%$ (table 4 ). These results were not statistically different from those obtained for comparison of the Chlamydia Rapid Test with polymerase chain reaction $(\mathrm{P}=0.317)$.

\section{Performance of the Chlamydia Rapid Test with asymptomatic patients}

At the time of recruitment to the study, the proportion of polymerase chain reaction positive participants without genitourinary symptoms was $98.2 \%$ at site 1 , $38.9 \%$ at site 2 , and $44.4 \%$ at site 3 . Of the asymptomatic patients detected by polymerase chain reaction assay, $83.6 \%$ at site $1,71.4 \%$ at site 2 , and $75 \%$ at site 3 were also detected by the Chlamydia Rapid Test, giving an overall Chlamydia Rapid Test sensitivity in asymptomatic women of $80.5 \%$.

\section{Organism load in polymerase chain reaction positive participants}

A second self collected vaginal swab specimen was available for participants at site 1 , which allowed the determination of the organism load of Chlamydia trachomatis in polymerase chain reaction positive participants. We analysed DNA extracted from the self collected vaginal swab specimens by quantitative polymerase chain reaction assay with a primer set that amplifies a highly conserved sequence of the $7.5 \mathrm{~kb}$ Chlamydia trachomatis cryptic plasmid. The organism load ranged from $5.97 \times 10^{2}$ to $1.09 \times 10^{9}$ Chlamydia trachomatis plasmids per swab (fig 2). Of the 56 polymerase chain reaction positive specimens from site 1 , only 33 had two matched swabs; of these, the Chlamydia trachomatis visual signal was significantly correlated with the Chlamydia trachomatis organism load in the samples tested $(r=0.644, \mathrm{P}<0.0001)$.

Acceptability of vaginal swab collection and test result waiting time

After specimen collection for the study was completed, we offered a written questionnaire to each participant.
The response rate was $80.3 \%(1083 / 1349)$; some of the returned questionnaires were not filled completely, so the total number of answers for each question varied slightly. The results showed that $99.4 \%(1072 / 1078)$ of respondents found the instructions easy to understand and $95.9 \%(1039 / 1083)$ felt comfortable collecting their own vaginal swab specimens. As to specimen preference, $40.7 \%(435 / 1068)$ preferred self collected vaginal swabs, whereas 37.5\% (401/1068) preferred urine, and the remaining 21.7\% (232/1068) did not show a preference for either sampling method (no significant difference among sites; $\mathrm{P}=0.069, \chi^{2}$ test). In terms of waiting time for the test result, $75.0 \%$ (661/ 881) of respondents indicated that they were willing to wait between 30 minutes and two hours for their test results. Other responses included less than 30 minutes, 6.9\% (61/881); more than two hours, $10.9 \%(96 / 881)$; and more than one day, $7.2 \%$ (63/ 881).

\section{DISCUSSION}

The results of this performance evaluation indicated that the new point of care Chlamydia Rapid Test could be used for diagnosis of chlamydial infection because of its good sensitivity and specificity. This new test provides a same day result, which would allow immediate treatment of the infected patient.

The Chlamydia Rapid Test was developed for the detection of Chlamydia trachomatis infection with noninvasive specimen types such as vaginal swabs. Unlike other rapid tests, the novel signal amplification system of the Chlamydia Rapid Test maximises the visual test signal, and the improved sample preparation chemistry overcomes signal inhibition caused by the high viscosity and variability of vaginal fluid. Specimen types for Chlamydia trachomatis testing have evolved in recent years, as studies have shown that vaginal specimens perform as well as, if not better than, endocervical swabs or first void urine across a range of nucleic acid amplification tests. ${ }^{18}{ }^{19}$ Vaginal swabs contain a higher load of Chlamydia trachomatis than does urine in women, providing another diagnostic advantage. ${ }^{14}$ The participants in our study indicated that they preferred vaginal swabs to urine because they did not have to wait for two hours since last voiding to collect their samples. Given the high acceptability of self collected vaginal swab sampling and the higher organism load in vaginal swabs than in urine, the self collected vaginal swab is the preferred specimen type for the Chlamydia Rapid Test.

This is the first published performance analysis for a Conformité Européenne licensed rapid test for Chlamydia with a claim for vaginal swab specimens. Earlier rapid tests for Chlamydia have shown poor sensitivity when compared with nucleic acid amplification tests using endocervical swab specimens. ${ }^{2021}$ A recent World Health Organization study of the Clearview Chlamydia MF test (Clearview, Inverness Medical, Bedford, UK) with cervical and vaginal swab specimens in China found its sensitivity versus polymerase chain reaction to be only $32.8 \%$ with vaginal swabs and 
$49.7 \%$ with endocervical swabs. ${ }^{22}$ Despite its rapid test format and minimal instrumentation requirements, these results suggest that the sensitivity of the Clearview test is unacceptably low.

The Chlamydia Rapid Test showed an overall resolved sensitivity of $83.5 \%$ and a positive predictive value of $86.7 \%$ with the participants in this study, who were mostly asymptomatic women. The performance of the Chlamydia Rapid Test did not differ significantly among the three clinical sites, suggesting that the reproducibility and robustness of the assay are high.

With the Chlamydia Rapid Test, results are available within 30 minutes, allowing all patients testing positive to be offered treatment while still at the clinic. Given that about 3\% of women diagnosed with Chlamydia infection have been found to develop pelvic inflammatory disease in the interval between testing and their return for treatment, ${ }^{23}$ the prompt treatment of infected women made possible by the Chlamydia Rapid Test would be expected to avert this outcome. A test and treat strategy might also help to prevent onward transmission of Chlamydia by sexual contact that occurs during the interval between standard testing and treatment. ${ }^{24}$ Tracing of contacts or notification of partners could also be started immediately, enabling more rapid testing and treatment of sexual partners. The lower sensitivity of the Chlamydia Rapid Test compared with nucleic acid amplification tests is thus counterbalanced by the immediate clinical care available to the patient and the potential public health benefits of earlier intervention. The results of this study further show the applicability of the "rapid test paradox" described by Gift and colleagues, ${ }^{25}$ whereby a rapid test with lower sensitivity allows for treatment of more infected patients because results are available before they leave the clinic.

A test with the characteristics of the Chlamydia Rapid Test could be a valuable addition to screening programmes for Chlamydia, given that the non-invasive specimen type and immediate results might be more attractive to young women than currently available nucleic acid amplification testing algorithms. This is especially true in low prevalence settings, where the Chlamydia Rapid Test can be used in upfront testing that is coupled with back-end testing of pooled urine with nucleic acid amplification. Several studies have evaluated and confirmed the utility, cost effectiveness, and accuracy of pooling urogenital specimens for nucleic acid amplification tests, particularly for laboratories with low prevalence of chlamydia. ${ }^{26-29}$ By combining the rapid result from the Chlamydia Rapid Test of individual specimens and confirming those that were missed by the Chlamydia Rapid Test through nucleic acid amplification testing of pooled samples, at least $83 \%$ of Chlamydia trachomatis infected patients could be treated immediately, without having to wait days for the nucleic acid amplified test result. The Chlamydia Rapid Test could also be applied to novel settings such as mobile clinics, outreach settings, and home self testing to help to improve the screening coverage of difficult to reach populations.
In clinical settings of developing countries, especially those with high risk populations such as female sex workers, the availability of the Chlamydia Rapid Test would also allow more people to be screened and treated. In these settings, Vickerman and colleagues estimated that a point of care test for Chlamydia of "moderate sensitivity" could lead to the detection and treatment of substantially more infections than would the gold standard test. ${ }^{30}$ With the health resource constraints of most developing nations, the Chlamydia Rapid Test could be implemented easily without the need for laboratory equipment or highly trained staff, as seen in a study in two resource limited clinics in the Philippines. ${ }^{31}$

\section{Strengths and weaknesses of the study}

The strengths of this study include its multicentre design and large sample sizes at both the low prevalence and high prevalence sites. Confirmatory testing was done on discordant samples to resolve true Chlamydia positivity, along with additional random testing to minimise the bias from "selective" analysis of discordant samples. The study also showed high reproducibility of interoperator testing, strengthening the validity of the laboratory methods.

Ideally, the comparator test should have been a Chlamydia trachomatis nucleic acid amplified test using endocervical swab specimens, but given that such swab collection was not available at one of the sites (site 1), we compared the performance of the Chlamydia Rapid Test with nucleic acid amplification testing of a specimen type (first void urine) that could be collected at all three sites. We circumvented this weakness by comparing the Chlamydia Rapid Test with the genitourinary medicine clinic test (that is, strand displacement amplification assay on endocervical swab specimens); no significant differences in sensitivity or specificity was detected for the Chlamydia Rapid Test with either polymerase chain reaction or strand displacement amplification assay as the comparator test.

\section{Future research}

Randomised controlled trials are needed to examine the effectiveness of both opportunistic and proactive chlamydia screening strategies that use both the Chlamydia Rapid Test and nucleic acid amplification tests, and thereby to determine the most appropriate and cost effective approaches for the use of these tests in different clinical settings. Chlamydia infections in patients with low organism loads are those most likely to elude detection with the Chlamydia Rapid Test. Given that the organism load of Chlamydia trachomatis in women is associated with multiple symptoms and clinical signs, ${ }^{24}$ further research is needed to determine the clinical significance and transmission dynamics of low load infection in both men and women.

\section{Conclusions}

The new Chlamydia Rapid Test evaluated in this study achieves relatively high diagnostic sensitivity and 


\section{WHAT IS ALREADY KNOWN ON THIS TOPIC}

Nucleic acid amplification tests for Chlamydia are more sensitive and specific than are currently available rapid tests, but they are unaffordable in resource limited clinics The turnaround time from testing to results of one to two weeks for nucleic acid amplification tests also precludes immediate instigation of treatment and notification of partners

\section{WHAT THIS STUDY ADDS}

A novel rapid test for Chlamydia can achieve a high level of sensitivity and specificity in women with the use of noninvasive vaginal swab specimens

The Chlamydia Rapid Test is thus a potential cost effective alternative to nucleic acid amplification tests for diagnosis of Chlamydia infection and in screening programmes It also has the potential to enhance Chlamydia control strategies by allowing testing with non-invasive specimens as well as allowing treatment to be started on the same day

provides results within about 30 minutes. It is suitable as a primary diagnostic tool for Chlamydia infection and, in settings where access to nucleic acid amplification tests is limited or absent, could also be used as a screening tool, especially for high risk populations. Further evaluation of the Chlamydia Rapid Test in different resource limited settings would provide information on the utility of this test, both as a diagnostic tool and as a screening tool for Chlamydia infection.

We thank I Clarke (University of Southampton) for providing the plasmid PCTL12A used as a standard in the quantitative analysis; I White (Guy's and S Thomas' Hospital, London), J-P Allain (University of Cambridge), and ECB Nadala Jr (Diagnostics for the Real World (Europe) Ltd) for critical review of the manuscript; and the participants and staff at the three clinical sites of the study.

Contributors: HHL was the chief investigator of the study. LM-T, VL, and HHL prepared the clinical plan and acted as clinical monitors. LM-T wrote the first draft of the manuscript. VL and JJW were responsible for data entry and analysis. BTG, IU-L, and PB were the principal investigators at the clinical sites. AS waS the clinician involved in the study at site 1 . Cl and SA tested and analysed discordant samples. All authors participated in drafting and revising the manuscript, and all approved the final version. HHL is the guarantor Funding: Wellcome Trust grant to the University of Cambridge and additional support from the NIHR Cambridge Biomedical Research Centre.

Competing interests: JJW and HHL are equity holders of a spin-off company, Diagnostics for the Real World, based on the rapid test technologies developed at the University of Cambridge. Both the University of Cambridge and the Wellcome Trust are also equity holders of the company. Ethical approval: Moorfields and Whittington Research Ethics Committee (05/Q0504/53); Brook in Birmingham Research Ethics Committee. Provenance and peer review:Not commissioned; externally peer reviewed.

1 Cates W, Wasserheit JN. Genital chlamydial infections: epidemiology and reproductive sequelae. Am J Obstetr Gynecol 1991;164:1771-81.

2 Paavonen J, Lehtinen M. Chlamydial pelvic inflammatory disease. Hum Reprod Update 1996;2:519-29.

3 Gaydos CA, Theodore M, Dalesio N, Wood BJ, Quinn TC. Comparison of three nucleic acid amplification tests for detection of Chlamydia trachomatis in urine specimens. J Clin Microbiol 2004;42:3041-5.

4 National Chlamydia Screening Programme Steering Group. New frontiers: annual report of the national chlamydia screening programme in England 2005/06. London: Health Protection Agency, 2006 (available at www.hpa.org.uk/publications/2006/ncsp/).

5 Davies SC, Otto B, Partohudoyo S, Chrisnadarmani VAMA, Neilsen GA, Ciaffi L, et al. Sexually transmitted infections among female sex workers in Kupang, Indonesia. Sex Transm Dis 2003;30:671-9.
6 Nessa K, Waris SA, Alam A, Huq M, Nahar S, Chawdhary FAH. Sexually transmitted infections among brothel-based sex workers in Bangladesh: high prevalence of asymptomatic infection. Sex Transm Dis 2005;32:13-9.

7 Wi T, Ramos ER, Steen R, Roces MCR, Lim-Quizon MC, Neilsen G, et al. STI declines among sex workers and clients following outreach, onetime presumptive treatment and regular screening of sex workers in the Philippines. Sex Transm Infect 2006;82:386-91.

8 Dallabeta GA, Gerbase AC, Holmes KK. Problems, solutions, and challenges in syndromic management of sexually transmitted diseases. Sex Transm Infect 1998;74(suppl 1):S1-11.

9 Shelton JD. Prevention first: a three-pronged strategy to integrate family planning programme efforts against HIV and sexually transmitted infections. Int Family Planning Persp 1999;25:127-52.

10 Moi H, Hartgill U. Handilab C Chlamydia Hometest: doesn't deliver what it promises [abstract]. Int J STD AIDS 2007;18(suppl 1):20-1.

11 National Committee on Clinical Laboratory Standards. User protocol for evaluation of qualitative test performance: approved guideline. No. EP12-A 2002;22(14).

12 Michel CE, Solomon AW, Magbanua JPV, Massae P, Huang L, Mosha J, et al. Field evaluation of a rapid point-of-care assay for targeting antibiotic treatment for trachoma control: a comparative study. Lancet 2006;367:1585-90.

13 Pickett MA, Everson S, Pead PJ, Clarke IN. The plasmids of Chlamydia trachomatis and Chlamydophila (N16): accurate determination of copy number and the paradoxical effect of plasmid-curing agents. Microbiology 2005;151:893-903.

14 Michel CE, Sonnex C, Carne CA, White JA, Magbanua JPVM, Nadala ECB, et al. Chlamydia trachomatis load at matched anatomic sites: implications for screening strategies. J Clin Microbiol 2007; 45:1395-402.

15 Pasternack R, Vuorinen P, Kuukankorpi A, Pitkajarvi T, Miettinen D. Detection of Chlamydia trachomatis infections in women by Amplicor PCR: comparison of diagnostic performance with urine and cervical specimens. J Clin Microbiol 1996;34:995-8.

16 Moncada J, Chow JM, Schachter J. Volume effect on sensitivity of nucleic acid amplification tests for detection of Chlamydia trachomatis in urine specimens from females. / Clin Microbiol 2003;41:4842-3.

17 Verkooyen AP, Noordhoek GT, Klapper PE, Reid J, Schirm J, Cleator GM, et al. Reliability of nucleic acid amplification methods for detection of Chlamydia trachomatis in urine: results of the first international collaborative quality control study among 96 laboratories. / Clin Microbiol 2003;41:3013-6.

18 Shafer M-A, Moncada J, Boyer CB, Betsinger K, Flinn SD, Schachter J. Comparing first-void urine specimens, self-collected vaginal swabs, and endocervical specimens to detect Chlamydia trachomatis and Neisseria gonorrhoeae by a nucleic acid amplification test. J Clin Microbiol 2003;41:4395-9.

19 Schachter J, McCormack WM, Chernesky M, Martin DH, Van der Pol B, Rice PA, et al. Vaginal swabs are appropriate specimens for diagnosis of genital tract infection with Chlamydia trachomatis. J Clin Microbiol 2003;41:3784-9.

20 Pate MS, Dixon PB, Hardy K, Crosby M, Hook III EW. Evaluation of the Biostar Chlamydia OIA assay with specimens from women attending a sexually transmitted disease clinic. J Clin Microbiol 1998;36:2183-6.

21 Lauderdale T-L, Landers L, Thorneycroft I, Chapin K. Comparison of the PACE 2 assay, two amplification assays and Clearview EIA for detection of Chlamydia trachomatis in female endocervical and urine specimens. J Clin Microbiol 1999;37:2223-9.

22 Yin Y-P, Peeling RW, Chen X-S, Gong K-L, Zhou H, Gu W-M, et al. Clinicbased evaluation of Clearview Chlamydia MF for detection of Chlamydia trachomatis in vaginal and cervical specimens from women at high risk in China. Sex Transm Infect 2006;82:33-7.

23 Hook EW III, Spitters C, Reichart CA, Neumann TM, Quinn TC. Use of cell culture and a rapid diagnostic assay for Chlamydia trachomatis screening. JAMA 1994;272:867-70.

24 Centers for Disease Control and Prevention. Sexually transmitted disease treatment guidelines 2002. MMWR Recomm Rep 2002;51:1-78

25 Gift TL, Pate S, Hook EW III, Kassler WJ. The rapid test paradox: when fewer cases detected lead to more cases treated: a decision analysis of tests for Chlamydia trachomatis. Sex Transm Dis 1999;26:232-40.

26 Peeling RW, Toye B, Jessamine P, Gemmill I. Pooling of urine specimens for PCR testing: a cost saving strategy for Chlamydia trachomatis control programmes. Sex Trans Infect 1998;74:66-70.

27 Krepel J, Patel J, Sproston A, Hopkins F, Jang D, Mahoney J, et al. The impact on accuracy and cost of ligase chain reaction testing by pooling urine specimens for the diagnosis of Chlamydia trachomatis infections. Sex Transm Dis 1999;26:504-7.

28 Morre SA, Meijer CILM, Munk C, Kruger-Kjaer S, Winther JF, Jorgensens $\mathrm{HO}$, et al. Pooling of urine specimens for detection of asymptomatic Chlamydia trachomatis infections by PCR in a low- 
prevalence population: cost-saving strategy for epidemiological studies and screening programs. J Clin Microbiol 2000;38:1679-80.

29 Currie MJ, McNiven M, Yee T, Schiemer U, Bowden FJ. Pooling of clinical specimens prior to testing for Chlamydia trachomatis by PCR is accurate and cost saving. J Clin Microbiol 2004;42:4866-7.

30 Vickerman P, Watts C, Alary M, Mabey D, Peeling RW. Sensitivity requirements for the point of care diagnosis of Chlamydia trachomatis and Neisseria gonorrhoea in women. Sex Transm Infect 2003;79:363-8.
31 Saison F, Mahilum-Tapay L, Michel CE, Buttress N, Nadala ECB, Magbanua JP, et al. Prevalence of Chlamydia trachomatis infection and performance of Chlamydia rapid tests among low- and high-risk Filipino women in resource-limited settings. J Clin Microbiol 2007 doi: 10.1128/JCM.01343-07.

Accepted: 9 October 2007 